\title{
PROGRESS IN M-THEORY*
}

\author{
M. J. DUFFt \\ Michigan Center for Theoretical Physics \\ Randall Laboratory, Department of Physics, University of Michigan \\ Ann Arbor, MI 48109-1120, USA
}

\begin{abstract}
After reviewing how M-theory subsumes string theory, we report on some new and interesting developments, focusing on the "brane-world": circumventing no-go theorems for supersymmetric brane-worlds and complementarity of the Maldacena and RandallSundrum pictures. We also discuss the quantum $M \rightarrow 0$ discontinuity of massive gravity with a A term.
\end{abstract}

My talk is in three parts. In section 1 we briefly review M-theory, in section 2 we discuss some recent developments on the brane-world and finally in section 3 we address the issue of whether the graviton can have a mass.

\section{The story so far}

\subsection{M-theory and dualities}

Not so long ago it was widely believed that there were five different superstring theories each competing for the title of "Theory of everything," that all-embracing theory that describes all physical phenomena. See Table 1.

Moreover, on the $(d, D)$ "branescan" of supersymmetric extended objects with $d$ worldvolume dimensions moving in a spacetime of $D$ dimensions, all these theories occupied the same $(d=2, D-10)$ slot. See table 2 . The orthodox wisdom was that while ( $d=2, D=10$ ) was the Theory of Everything, the other branes on the scan were Theories of Nothing. All that has now changed. We now know that there are not five different theories at all but, together with $D=11$ supergravity, they form merely six different corners of a deeper, unique and more profound theory called " $\mathrm{M}$ theory" where M stand for Magic, Mystery or Membrane. M-theory involves all of the other branes on the branescan, in particular the eleven-dimensional membrane $(\mathrm{d}=3, D=11)$ and eleven-dimensional fivebrane $(d=6, D=11)$, thus resolving the mystery of why strings stop at ten dimensions while supersymmetry allows eleven ${ }^{2}$.

Although we can glimpse various corners of M-theory, the big picture still eludes us. Uncompactified M-theory has no dimensionless parameters, which is good from the uniqueness point of view but makes ordinary perturbation theory impossible since there are no small coupling constants to provide the expansion parameters. A

*Research supported in part by DOB Grant DE-FG02-95ER40899.

† mduff@umich.edu 


\begin{tabular}{l|c|c|c} 
& Gauge Group & Chiral? & Supersymmetry charges \\
\hline Type I & $\mathbf{5 0}(\mathbf{3 2})$ & yes & $\mathbf{1 6}$ \\
\hline Type IIA & $U(1)$ & no & $\mathbf{3 2}$ \\
\hline Type IIB & - & yes & $\mathbf{3 2}$ \\
\hline Heterotic & $E_{8} \times E_{8}$ & yes & $\mathbf{1 6}$ \\
\hline Heterotic & $S O(32)$ & yes & $\mathbf{1 6}$ \\
\hline
\end{tabular}

Table 1: The Five Superstring Theories

low energy, $E$, expansion is possible in powers of $E / M_{P}$, with $M p$ the Planck mass, and leads to the familiar $D=11$ supergravity plus corrections of higher powers in the curvature. Figuring out what governs these corrections would go a long way in pinning down what M-theory really is.

Why, therefore, do we place so much trust in a theory we cannot even define? First we know that its equations (though not in general its vacua) have the maximal number of 32 supersymmetry charges. This is a powerful constraint and provides many "What else can it be?" arguments in guessing what the theory looks like when compactified to $D<11$ dimensions. For example, when M-theory is compactified on a circle $S^{1}$ of radius $R_{11}$, it leads to the Type $I I A$ string, with string coupling constant $g_{s}$ given by

$$
g_{s}=\widetilde{\kappa}_{11}^{3 / 2}
$$

We recover the weak coupling regime only when $R_{11} \rightarrow 0$, which explains the earlier illusion that the theory is defined in $D=10$. Similarly, if we compactifyon a line segment (known technically as $S^{1} / Z_{2}$ ) we recover the $E_{8} \times E_{8}$ heterotic string. Moreover, although the corners of M-theory we understand best correspond to the weakly coupled, perturbative, regimes where the theory can be approximated by a string theory, they are related to one another by a web of dualities, some of which are rigorously established and some of which are still conjectural but eminently plausible. For example, if we further compactify Type IIA string on a circle of radius $R$, we can show rigorously that it is equivalent to the Type $I I B$ string compactified on a circle or radius $1 / R$. If we do the same thing for the $E_{8} \times E_{8}$ heterotic string we recover the $S O(32)$ heterotic string. These well-established relationships which remain within the weak coupling regimes are called $T$-dualities. The name $S$-dualities refers to the less well-established strong/weak coupling relationships. For example, the $S O(32)$ heterotic string is believed to be $S$-dual to the $S O(32)$ Type / string, and the Type $I I B$ string to be self-S-dual. If we compactify more dimensions, other dualities can appear. For example, the heterotic string compactified on a six-dimensional torus $T^{6}$ is also believed to be self- $S$-dual. There is also the phenomenon of duality of dualities by which the $T$-duality of one theory is the $S$-duality of another. When M-theory is compactified on $T^{n}$, these $\mathrm{S}$ and $T$ dualities are combined into what are termed $U$-dualities. All the consistency checks we have been able to think of (and after 5 years there dozens of them) have worked and 


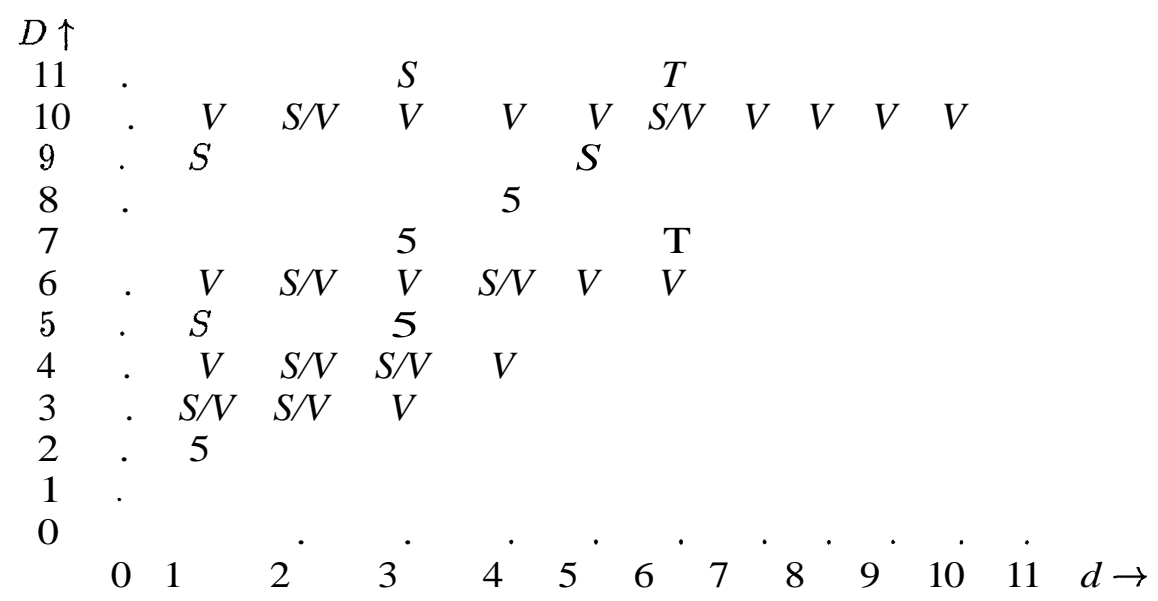

Table 2: The branescan, where 5, $V$ and $\mathrm{T}$ denote scalar, vector and antisymmetric tensor multiplets.

convinced us that all these dualities are in fact valid. Of course we can compactify M-theory on more complicated manifolds such as the four-dimensional $K 3$ or the six-dimensional Calabi-Yau spaces and these lead to a bewildering array of other dualities. For example: the heterotic string on $T^{4}$ is dual to the Type // string on $K 3$; the heterotic string on $T^{6}$ is dual to the the Type // string on Calabi-Yau; the Type IIA string on a Calabi-Yau manifold is dual to the Type IIB string on the mirror Calabi-Yau manifold. These more complicated compactifications lead to many more parameters in the theory, known to the mathematicians as moduli, but in physical uncompactified spacetime have the interpretation as expectation values of scalar fields. Within string perturbation theory, these scalar fields have flat potentials and their expectation values are arbitrary. So deciding which topology Nature actually chooses and the values of the moduli within that topology is known as the vacuum degeneracy problem.

\subsection{Branes}

In the previous section we outlined how M-theory makes contact with and relates the previously known superstring theories, but as its name suggest, M-theory also relies heavily on membranes or more generally $p$-branes, extended objects with $p=d-1$ spatial dimensions (so a particle is a 0-brane, a string is a 1-brane, a membrane is a 2-brane and so on). In $D=4$, a charged 0-brane couples naturally to an Maxwell vector potential $A_{\mu}$, with field strength $F_{\mu \nu}$ and carries an electric charge

$$
Q \sim \underset{J_{S}}{I_{2}} * F_{2}
$$


and magnetic charge

$$
P \sim \int_{S^{2}} F_{2}
$$

where $F_{2}$ is the Maxwell 2-form field strength, $* F_{2}$ is its 2 -form dual and $S^{2}$ is a 2 -sphere surrounding the charge. This idea may be generalized to $p$-branes in $D$ dimensions. A $p$-brane couples to $(p+1)$-form potential $A_{\mu_{1} \mu_{2} \ldots \mu_{p+1}}$ with $(P+2)$ form field strength $F_{\mu_{1} \mu_{2} \ldots \mu_{p+2}}$ and carries an "electric" charge per unit p-volume

$$
Q \sim \int_{S^{D-p-2}} * F_{D-p-2}
$$

and "magnetic" charge per unit p-volume

$$
P \sim \int_{S^{p+2}} F_{p+2}
$$

where $F_{p+2}$ is the $(p+2)$-form field strength, $* F_{D-p-2}$ its $(D-p-2)$-form dual and $S^{n}$ is an n-sphere surrounding the brane. A special role is played in $M$-theory by the BPS (Bogomolnyi-Prasad-Sommerfield) branes whose mass per unit $p$-volume, or tension $T$, is equal to the charge per unit $p$-volume

$$
T \sim Q
$$

This formula may be generalized to the cases where the branes carry several electric and magnetic charges. The supersymmetric branes shown on the branescan are always BPS, although the converse is not true. M-theory also makes use of non-BPS and non-supersymmetric branes not shown on the branescan, but the supersymmetric ones do play a special role because they are guaranteed to be stable.

The letters $\mathrm{S}, \mathrm{V}$, and $\mathrm{T}$ on the branescan refer to scalar, vector and antisymmetric tensor supermultiplets of fields that propagate on the worldvolume of the brane. Historically, these points on the branescan were discovered in three different ways. The $\mathrm{S}$ branes were classified by writing down spacetime supersymmetric worldvolume actions that generalize the Green-Schwarz actions on the superstring worldsheet ${ }^{5}$. By contrast, the $\mathrm{V}$ and $\mathrm{T}$ branes were shown to arise as soliton solutions ${ }^{a}$ of the underlying supergravity theories ${ }^{6}$. However, the solitonic $\mathrm{V}$ branes found this way were bound by $\mathrm{p}<7$. The 8 -brane and 9-brane slots were included on the scan only because they were allowed by supersymmetry ${ }^{6}$. Subsequently, all the V-branes were given a new interpretation as Dirichlet p-branes, called Dbranes, surfaces of dimension $\mathrm{p}$ on which open strings can end and which carry R-R (Ramond-Ramond) charge ${ }^{9}$. The IIA theory has D-branes with $\mathrm{p}-0,2,4,6,8$ and the IIB theory has D-branes with $\mathrm{p}=1,3,5,7,9$. They are related to one another by $\mathrm{T}$-duality. In terms of how their tensions depend on the string coupling $g_{s}$, the

${ }^{a}$ The 3-brane soliton of Type IIB supergravity was an early candidate for a 'brane-world', firstly because of its dimensionality ${ }^{7,8}$ and secondly because gauge fields propagate on its worldvolume ${ }^{8}$. See section 2.2. 
D-branes are mid-way between the fundamental (F) strings and the solitonic (S) fivebranes:

$$
T_{F 1} \sim m_{s}{ }^{2}, \quad T_{D p} \sim \frac{m_{s}{ }^{p+1}}{9 s}, \quad T_{S 5} \sim \frac{m_{s}{ }^{6}}{9 s^{2}}
$$

Since they are BPS, there is a no-force condition between the branes that allows us to have many branes of the same charge parallel to one another. The gauge group on a single D-brane is an abelian $U(1)$. If we stack $N$ such branes on top of one another, the gauge group is the non-abelian $U(N)$. As we separate them this decomposes into its subgroups, so in fact there is a Higgs mechanism at work whereby the vacuum expectation values of the Higgs fields are related to the separation of the branes. For example the theory that lives on a stack of $N$ Type IIB $D 3$ branes is a four-dimensional $U(N) n-4$ super Yang-Mills theory. In the limit of large $N$ the geometry of this configuration tends to the product of five dimensional anti-de Sitter space and a five dimensional sphere, $A d S_{5} \times S^{5}$.

In $D=11$, M-theory has two BPS branes, an electric 2-brane and its magnetic dual which is a 5-brane. Their tensions are related to each other and the Planck mass by

$$
T_{2}^{3} \sim T_{5} \sim M_{P}^{6}
$$

if we stack $N$ such branes on top of one another, the M2-brane geometry tends in the large $N$ limit to $A d S_{4} \times S^{7}$ and the M5-brane geometry to $A d S_{7} \times S^{4}$. In addition there are two other objects in $D=11$, the plane wave and the Kaluza-Klein monopole, which though not branes are still BPS. When spacetime is compactified a $p$-brane may remain a $p$-brane or else become a $(p-k)$-brane if it wraps around $k$ of the compactified directions. For example, the Type $I I A$ fundamental string emerges by wrapping the M2-brane around $S^{1}$ and shrinking its radius to zero, and the Type II $A$ 4-brane emerges in a similar way from the $M 5$-brane.

\subsection{Spin-offs of M-theory}

What do we now know with M-theory that we did not know with old-fashioned string theory? Here are a few examples, references to which may be found in Ref. 2.

1) Electric-magnetic (strong/weak coupling) duality in $D=4$ is a consequence of string/string duality in $D=6$ which in turn is a consequence of membrane/fivebrane duality in $D=11$.

2) Exact electric-magnetic duality, first proposed for the maximally supersymmetric conformally invariant $n=4$ super Yang-Mills theory, has been extended to effectiveduality by Seiberg and Witten to non-conformal $n=2$ theories: the so-called Seiberg-Witten theory. This has been very successful in providing the first proofs of quark confinement (albeit in the as-yet-unphysical super QCD) and in generating new pure mathematics on the topology of four-manifolds. SeibergWitten theory and other $n=1$ dualities of Seiberg may, in their turn, be derived from M-theory.

3) Indeed, it seems likely that all supersymmetric quantum field theories with any gauge group, and their spontaneous symmetry breaking, admit a geometrical 
interpretation within M-theory as the worldvolume fields that propagate on the common intersection of stacks of p-branes wrapped around various cycles of the compactified dimensions, with the Higgs expectation values given by the brane separations.

4) In perturbative string theory, the vacuum degeneracy problems arises because there are billions of Calabi-Yau vacua which are distinct according to classical topology. Like higher-dimensional Swiss cheeses, each can have differentnumber of $p$-dimensional holes. This results in many different kinds of four-dimensional gauge theories with different gauge groups, numbers of families and different choices of quark and lepton representations. Moreover, M-theory introduces new nonperturbative effects which allow many more possibilities, making the degeneracy problem apparently even worse. However, most (if not all) of these manifolds are in fact smoothly connected in M-theory by shrinking the $p$-branes that can wrap around the $p$-dimensional holes in the manifold and which appear as black holes in spacetime. As the wrapped-brane volume shrinks to zero, the black holes become massless and effect a smooth transition from one Calabi-Yau manifold to another. Although this does not yet cure the vacuum degeneracy problem, it puts it in a different light. The question is no longer why we live in one topology rather than another but why we live in one particular corner of the unique topology. This may well have a dynamical explanation.

5) Ever since the 1970's, when Hawking used macroscopic arguments to predict that black holes have an entropy equal to one quarter the area of their event horizon, a microscopic explanation has been lacking. But treating black holes as wrapped $p$ branes, together with the realization that Type II branes have a dual interpretation as Dirichlet branes, allows the first microscopic prediction in complete agreement with Hawking. The fact that $\mathrm{M}$-theory is clearing up some long standing problems in quantum gravity gives us confidence that we are on the right track.

6) It is known that the strengths of the four forces change with energy. In supersymmetric extensions of the standard model, one finds that the fine structure constants $\alpha_{3}, \alpha_{2}, \alpha_{1}$ associated with the $S U(3) \times 5(7(2) \times(7(1)$ all meet at about $10^{16} \mathrm{GeV}$, entirely consistent with the idea of grand unification. The strength of the dimensionless number $\alpha_{G}-G E^{2}$, where $G$ is Newton's constant and $E$ is the energy, also almost meets the other three, but not quite. This near miss has been a source of great interest, but also frustration. However, in a universe of the kind envisioned by Witten, spacetime is approximately a narrow five dimensional layer bounded by four-dimensional walls. The particles of the standard model live on the walls but gravity lives in the five-dimensional bulk. As a result, it is possible to choose the size of this fifth dimension so that all four forces meet at this common scale. Note that this is much less than the Planck scale of $10^{19} \mathrm{GeV}$, so gravitational effects may be much closer in energy than we previously thought; a result that would have all kinds of cosmological consequences.

So what is M-theory?

There is still no definitive answer to this question, although several different 
proposals have been made. By far the most popular is $\mathrm{M}$ (atrix) theory ${ }^{10}$. The matrix models of $M$-theory are $U(N)$ supersymmetric gauge quantum mechanical models with 16 supersymmetries. Such models are also interpretable as the effective action of $N$ coincident Dirichlet 0-branes.

The theory begins by compactifying the eleventh dimension on a circle of radius $R$, so that the longitudinal momentum is quantized in units of $1 / R$ with total $P L$ $N / R$ with $N \rightarrow \infty$. The theory is holographic in that it contains only degrees of freedom which carry the smallest unit of longitudinal momentum, other states being composites of these fundamental states. This is, of course entirely consistent with their identification with the Kaluza-Klein modes. It is convenient to describe these $N$ degrees of freedom as $N$ x $N$ matrices. When these matrices commute, their simultaneous eigenvalues are the positions of the 0-branes in the conventional sense. That they will in general be non-commuting, however, suggests that to properly understand $M$-theory, we must entertain the idea of a fuzzy spacetime in which spacetime coordinates are described by non-commuting matrices. In any event, this matrix approach has had success in reproducing many of the expected properties of M-theory such as $D=11$ Lorentz covariance, $D=11$ supergravity as the low-energy limit, and the existence of membranes and fivebranes.

It was further proposed that when compactified on $T^{d-1}$, the quantum mechanical model should be replaced by an $d$-dimensional $U(N)$ Yang-Mills field theory defined on the dual torus $\tilde{T}^{d-1}$. Another test of this M(atrix) approach, then, is that it should explain the $U$-dualities. For $d=4$, for example, this group is $S L(3, Z) \times S L(2, Z)$. The $S L(3, Z)$ just comes from the modular group of $T^{3}$ whereas the $S L(2, Z)$ is the electric/magnetic duality group of four-dimensional $n=4$ Yang-Mills. For $d>4$, however, this picture looks suspicious because the corresponding gauge theory becomes non-renormalizable and the full $U$-duality group has still escaped explanation. There have been speculations on what compactified M-theory might be, including a revival of the old proposal that it is really M(embrane)theory. In other words, perhaps $D=11$ supergravity together with its BPS configurations: plane wave, membrane, fivebrane, KK monopole and the $D=11$ embedding of the Type $I I A$ eightbrane, are all there is to M-theory and that we need look no further for new degrees of freedom, but only for a new nonperturbative quantization scheme. At the time of writing this is still being hotly debated.

What seems certain, however, is that M-theory is not a string theory. It can be approximated by a string theory only in certain peculiar corners of parameter space. So "string phenomenology" will become an oxymoron unless, for some as yet unknown reason, our universe happens to occupy one of these corners.

\subsection{AdS/CFT and the brane-world}

The year 1998 marked a renaissance in anti de-Sitter space (AdS) brought about by Maldacena's conjectured duality between physics in the bulk of $A d S$ and a conformal field theory on its boundary ${ }^{11}$. For example, M-theory on $A d S_{4} \times S^{7}$ is dual to 
a non-abelian ( $n=8, d=3$ ) superconformal theory, Type $I I B$ string theory on $A d S_{5} \times S^{5}$ is dual to a $(n=4, d=4) U(N)$ super Yang-Mills theory and $M$-theory on $A d S_{7} \times S^{4}$ is dual to a non-abelian $\left(\left(n_{+}, n_{-}\right)=(2,0), d=6\right)$ conformal theory. In particular, as has been spelled out most clearly in the $d=4 U(N)$ Yang-Mills case, there is seen to be a correspondence between the Kaluza-Klein mass spectrum in the bulk and the conformal dimension of operators on the boundary ${ }^{12,13}$. We note that, by choosing Poincare coordinates on $\mathrm{AdS}_{5}$, the metric may be written as

$$
d s^{2}=e^{-2 y / L}\left(d x^{\mu}\right)^{2}+d y^{2},
$$

where $x^{\mu},(\mu=0,1,2,3)$, are the four-dimensional brane coordinates. In this case the superconformal Yang-Mills theory is taken to reside at the boundary $y-\rightarrow-\infty$. The AdS length scale $L$ is given by

$$
L^{4}=4 \pi \alpha^{2}\left(g_{Y M}^{2} N\right)
$$

The string coupling $g_{s}$ and the Yang-Mills coupling $g_{Y M}$ are related by

$$
g_{s}=g_{Y M}{ }^{2}
$$

The full quantum string theory on this spacetime is difficult to deal with, but we can approximate it by classical Type IIB supergravity provided

$$
L^{2}>>\alpha^{\prime} \quad(12)
$$

so that stringy correction to supergravity are small, and that $g_{s}<<1$ or

$$
N \rightarrow \infty \quad(13)
$$

so that loop corrections can be neglected. There is now overwhelming evidence in favor of this correspondence and it allows us to calculate previously uncalculable strong coupling effects in the gauge theory starting from classical supergravity. Models of this kind, where a bulk theory with gravity is equivalent to a boundary theory without gravity, have also been advocated by 't Hooft ${ }^{14}$ and by Susskind ${ }^{15}$ who call them holographic theories. Many theorists are understandably excited about the AdS/CFT correspondence because of what it can teach us about nonperturbative QCD. In my opinion, however, this is, in a sense, a diversion from the really fundamental question: What is M-theory? So my hope is that this will be a two-way process and that superconformal field theories will also teach us more about M-theory.

The Randall-Sundrum mechanism ${ }^{16}$ also involves AdS but was originally motivated, not via the decoupling of gravity from D3-branes, but rather as a possible mechanism for evading Kaluza-Klein compactification by localizing gravity in the presence of an uncompactified extra dimension. This was accomplished by inserting a positive tension 3-brane (representing our spacetime) into $\mathrm{AdS}_{5}$. In terms of the Poincare patch of $\mathrm{AdS}_{5}$ given above, this corresponds to removing the region $y<0$, 
and either joining on a second partial copy of $\mathrm{AdS}_{5}$, or leaving the brane at the end of a single patch of $\mathrm{AdS}_{5}$. In either case the resulting Randall-Sundrum metric is given by

$$
d s^{2}=e^{-2|y| / L}\left(d x^{\mu}\right)^{2}+d y^{2},
$$

where $y £(-\infty, \infty)$ or $y £[0, \infty)$ for a 'two-sided' or 'one-sided' Randall-Sundrum brane respectively.

The similarity of these two scenarios led to the notion that they are in fact closely tied together. To make this connection clear, consider the one-sided RandallSundrum brane. By introducing a boundary in $\mathrm{AdS}_{5}$ at $y=0$, this model is conjectured to be dual to a cutoff CFT coupled to gravity, with $y=0$, the location of the Randall-Sundrum brane, providing the UV cutoff. This extended version of the Maldacena conjecture ${ }^{17}$ then reduces to the standard AdS/CFT duality as the boundary is pushed off to $y \rightarrow-\infty$, whereupon the cutoff is removed and gravity becomes completely decoupled. Note in particular that this connection involves a single CFT at the boundary of a single patch of $\mathrm{AdS}_{5}$. For the case of a brane sitting between two patches of $\mathrm{AdS}_{5}$, one would instead require two copies of the CFT, one for each of the patches. A crucial test of this assumed complementarity of the Maldacena and Randall-Sundrum pictures is that both should yield the same corrections to Newton's law. See section 2.3.

A third development in the brane-world has been the idea that the extra dimensions are compact but much larger than the conventional Planck sized dimensions in traditional Kaluza-Klein theories ${ }^{18}$. This is possible if the standard model fields are confined to the $d=4$ brane with only gravity propagating in the $d>4$ bulk $^{18}$. We shall not pursue this possibility here.

\section{Developments on the Brane-World}

\subsection{No-go theorems for supersymmetry}

If we are to give a "top-down" justification of the Randall-Sundrum brane-world by embedding it in string theory or M-theory, it is desirable that the R-S picture be consistent with supersymmetry. Indeed, such a supersymmetric brane-world is necessary if the Maldacena and Randall-Sundrum (R-S) pictures are to stand any chance of being complementary. At first, however, this seemed to be problematical and several papers appeared in the literature suggesting that R-S could not be supersymmetric. Some of these no-go theorems listed below are exactly as they appeared; with others I have taken the liberty of setting up the straw man so as more effectively to knock him down.

1) R-S branes cannot be SUSY because massless supergravity scalars give kink-up and not kink-down potentials which do not bind gravity to the brane.

2) R-S branes cannot be SUSY because their tension is not that of a BPS brane. 
3) R-S branes cannot be SUSY because $\delta$-functions are incompatible with susy transformation rules.

4) R-S branes cannot be SUSY because the photon superpartners of the graviton cannot be bound to the brane.

\subsection{Yes-go theorems for Supersymmetry of the brane-world}

In fact, the domain-wall solution of Bremer et $\mathrm{al}^{19}$ provides a supersymmetric Type IIB Randall-Sundrum realization ${ }^{20}$. See also Refs. 21 and 22. So it is instructive to see how the no-go theorems are circumvented:

1) The required supergravity scalar is massive, being the breathing mode of the $S^{5}$ compactification $^{20}$. So the negative conclusions about massless scalars in Refs. 23,24, while correct, are not relevant.

2) The tension comes from two sources: the BPS D3-branes and the $\mathbf{k i n k}^{25}$. So the observation of Ref. 26 that the D3 brane tension is only $2 / 3$ of the $\mathrm{R}-\mathrm{S}$ tension, while correct, is not relevant.

3) The sign flip of the coupling constant across the brane removes the $\delta$-functions in the supersymmetry transformation rules ${ }^{27,28,29,20,30}$. So the problems raised by Ref. 31, while correct, are not relevant.

4) Photons can be bound to the brane but their bulk origin is not Maxwell's equations but rather odd-dimensional self-duality equations ${ }^{32,33}$. So the result of Ref. 37, that photons obeying Maxwell's equations in the bulk cannot be bound to the brane, while correct, is not relevant ${ }^{b}$

An entirely different question is whether a smooth domain wall can provide a supersymmetric Randall-Sundrum realization, and here ordinary supergravity seems to fail requiring some kind of higher derivative and presumably stringy corrections $^{23,38}$.

\subsection{Complementarity of the Maldacena and Randall-Sundrum pictures}

In his $1972 \mathrm{PhD}$ thesis under Abdus Salam, the author showed that, when oneloop quantum corrections to the graviton propagator are taken into account, the inverse square $r^{-2}$ behavior of Newton's gravity force law receives an $r^{-4}$ correction whose coefficient depends on the number and spins of the elementary particles ${ }^{39,40}$. Specifically, the potential looks like

$$
V(r)=\frac{G M}{r}\left(1+\frac{\alpha G}{r^{2}}\right)
$$

where $G$ is the four-dimensional Newton's constant, $\hbar=c=1$ and a is a purely numerical coefficientgiven, in the case of spins $s \leq 1$, by $45 \pi \alpha=12 N_{1}+3 N_{1 / 2}+N_{0}$, where $N_{s}$ are the numbers of particle species of spin $s$ going around the loop.

${ }^{b}$ The authors of Refs. 34,35,36 showed that, treated as test particles, Maxwell photons could be bound to the brane but their charge would be screened. However, the combined bulk EinsteinMaxwell equations rule out photons on the brane altogether ${ }^{37}$. 
Now fast-forward to 1999 when Randall and Sundrum proposed that our fourdimensional world is a 3-brane embedded in an infinite five-dimensional universe. Gravity reaches out into the five-dimensional bulk but the other forces are confined to the four-dimensional brane. Contrary to expectation, they showed that an inverse square $r^{-2}$ law for gravity is still possible but with an $r^{-4}$ correction coming from the massive Kaluza-Klein modes whose coefficient depends on the bulk cosmological constant. Their potential looks like

$$
V(r)=\frac{G M}{r}\left(1+\frac{2 L^{2}}{3 r^{2}}\right) .
$$

where $L$ is the radius of $\mathrm{AdS}_{5}$. Since (15) was the result of a four-dimensional quantum calculation and (16) the result of a five-dimensional classical calculation, they seem superficially completely unrelated. However, Ref. 41 invokes the AdS/CFT correspondence of Maldacena to demonstrate that the two are in fact completely equivalent ways of describing the same physics. From (15), we see that the contribution of a single $n=4 U(N)$ Yang-Mills CFT, with $\left(N_{1}, N_{1 / 2}, N_{0}\right)=\left(N^{2}, 4 N^{2}, 6 N^{2}\right)$, is

$$
V(r)=\frac{G M}{r}\left(1+\frac{2 N^{2} G}{3 \pi r^{2}}\right)
$$

Using the AdS/CFT relation $N^{2}=\pi L^{3} / 2 G_{5}$ and the one-sided brane-world relation $G=2 G_{5} / L$, where $G_{5}$ is the five-dimensional Newton's constant, we obtain exactly (16).

As discussed in the August 2000 edition of Scientific American ${ }^{18}$, experimental tests of deviations from Newton's inverse square law are currently under way.

\subsection{Five versus eleven}

As we have seen M-theory requires eleven dimensions, whereas if the brane-world picture is correct, we really need only five with the other six going along for the ride. Why should Nature behave like this? The only good answer to this question I could find is in Mother Goose's Nursery Rhymes:

Nature requires five,

Custom allows seven,

Idleness takes Nine,

And Wickedness Eleven.

\section{A massive graviton?}

An old question is whether the graviton has exactly zero mass or perhaps a small but non-zero mass. This issue seemed to have been resolved by van Dam and Veltman ${ }^{42}$ and, independently, Zakharov ${ }^{43}$ when they noted that there is a discrete difference between the propagator of a strictly massless graviton and that of a graviton with mass $M$ in the $M-\rightarrow 0$ limit. This difference gives rise to a discontinuity between the corresponding amplitudes involving graviton exchange. 
In particular, the bending of light by the sun in the massive case is only $3 / 4$ of the experimentally confirmed massless case, thus ruling out a massive graviton.

Recently, however, the masslessness of the graviton has been called into question by two papers ${ }^{44,45}$ pointing out that the van Dam-Veltman-Zakharov discontinuity disappears if, instead of being Minkowski, the background spacetime is anti-de Sitter (AdS). The same result in de Sitter space had earlier been obtained in Ref.46,47. In fact, as shown in Ref.48, this can be extended to any Einstein space satisfying

$$
R_{\mu \nu}=\Lambda g_{\mu \nu}
$$

with a non-zero cosmological constant A $\neq 0$ provided $M^{2} / \Lambda \rightarrow 0$.

Let us define the second-order spin operators acting on $(A, B)$ representations of the Lorentz group ${ }^{49}$ : the scalar Laplacian $\Delta(0,0)=-\square$, the Lichnerowicz operator for symmetric rank-2 tensors $\mathrm{A}(1,1) \phi_{\mu \nu}=-\square \phi+R_{\mu \tau} \phi_{\nu}^{\tau}+R_{\nu \tau} \phi_{\mu}^{\tau}-2 R_{\mu \rho \nu \tau} \phi^{\rho \tau}$. and the second-order vector operator by $\mathrm{A}(1 / 2,1 / 2) \xi_{\mu}=-\square \xi_{\mu}+R_{\mu \nu} \xi^{\nu}$. We have exploited the Einstein condition (18) for the background metric. Then one finds for $M \neq 0$, that the one-graviton exchange amplitude is given by

$$
\begin{aligned}
A[T]= & { }_{4\llcorner}\left[T^{\mu \nu}\left(\Delta(1,1)-2 \Lambda+M^{2}\right)^{-1} T_{\mu \nu}\right. \\
& \left.-\left(\frac{-2 \Lambda+2 M^{2}}{-2 \Lambda+3 M^{2}}\right) T_{\mu}^{\mu}\left(\Delta(0,0)-2 \Lambda+M^{2}\right)^{-1} T_{\mu}^{\mu}\right],
\end{aligned}
$$

in agreement with the result of Ref. 45, while for $M=0$

$$
\begin{aligned}
A[T]= & { }_{4\llcorner}\left[T^{\mu \nu}(\Delta(1,1)-2 \Lambda)^{-1} T_{\mu \nu}\right. \\
& \left.-T_{\mu}^{\mu}(\Delta(0,0)-2 \Lambda)^{-1} T_{\mu}^{\mu}\right],
\end{aligned}
$$

So for $\mathrm{A}=0$ there is a discontinuity as $M-\rightarrow 0$, but for $\mathrm{A} \neq 0$ the limit is continuous. These results remain surprising, however, since the massive graviton retains five degrees of freedom, while the massless one only has two. Although these extra states decouple from a covariantly conserved stress tensor for $M^{2} / \Lambda \rightarrow 0$, yielding a smooth classical limit, they are nevertheless still present in the theory, suggesting that a discontinuity may remain at the quantum level. In Ref.48, we demonstrate that this is indeed the case by calculating the one loop graviton vacuum amplitude for a massive graviton and showing that it does not reproduce the result for the massless case in the limit. Thus the apparent absence of the discontinuity is only an artifact of the tree approximation and the discontinuity reappears at one loop.

For $\mathrm{M} \neq 0$, the one-loop effective action $\Gamma$ is given by

$$
\exp (-\Gamma)=\frac{\left[\operatorname{det}\left(\Delta(1 / 2,1 / 9)-9 \Lambda+M^{2}\right)\right]^{1 / 2}}{[\operatorname{det}(\Delta(1,-}
$$

while for the $\mathrm{M}=0$ case

$$
\exp (-\Gamma)=\begin{gathered}
\operatorname{det}(\Delta(1 / 2,1 / 2)-2 \Lambda) \\
{[\operatorname{det}(\Delta(1,1)-2 \Lambda) \operatorname{det}(\Delta(0,0)-2 \Lambda)]^{1 / 2}}
\end{gathered}
$$


The difference in these two expressions reflects the fact that 5 degrees of freedom are being propagated around the loop in the massive case and only 2 in the massless case. Denoting the dimension of the spin $(A, B)$ representation by $D(A, B)=$ $(2 A+1)(2 B+1)$, we count $D(1,1)-D(1 / 2,1 / 2)=5$ for the massive case, while $D(1,1)-2 D(1 / 2,1 / 2)+D(0,0)=2$ for the massless one.

It remains to check that there is no conspiracy among the eigenvalues of these operators that would make these two expressions coincide. To show this, it suffices to calculate the coefficients in the heat-kernel expansion for the massive graviton propagator, and compare it with the massless case given in Ref. 49. The coefficient functions $b_{k}^{\left({ }^{\wedge}\right)}$ in the expansion

$$
\operatorname{Tr} e^{-\Delta^{(\Lambda)} t}=\sum_{k=0}^{\infty} t^{(k-4) / 2} I \delta^{4} x \sqrt{g} b_{k}^{(\Lambda)}
$$

were calculated in Ref. 49 for general "spin operators" $\Delta^{(\Lambda)}(A, B)=\Delta(A, B)-2 \mathrm{~A}$ with the result

$$
\begin{aligned}
180(4 \pi)^{2} b_{4}^{(\Lambda)}(1,1) & =189 R_{\mu \nu \rho \sigma} R^{\mu \nu \rho \sigma}-756 \Lambda^{2}, \\
180(4 \pi)^{2} b_{4}^{(\Lambda)}(1 / 2,1 / 2) & =-11 R_{\mu \nu \rho \sigma} R^{\mu \nu \rho \sigma}+984 \Lambda^{2}, \\
180(4 \pi)^{2} b_{4}^{(\Lambda)}(0,0) & =R_{\mu \nu \rho \sigma} R^{\mu \nu \rho q} 636 \Lambda^{2} .
\end{aligned}
$$

It is straightforward to extend those results to relevant massive operators $\Delta^{(\Lambda, M)}(A, B) \equiv$ $A(A, B)-2 \mathrm{~A}+M^{2}$. The coefficients $b_{k}^{(\Lambda, M)}(A, B)$ for these operators are perfectly smooth functions of $M^{2}$. Thus, as $M^{2} \rightarrow 0$, we obtain

$$
\begin{aligned}
180(4 \pi)^{2} b_{4}^{(\Lambda, M)}(\text { total }) & \\
& =180(4 \pi)^{2}\left[b_{4}^{(\Lambda, M)}(1,1)-b_{4}^{(\Lambda, M)}(1 / 2,1 / 2)\right] \\
& \rightarrow 200 R_{\mu \nu \rho \sigma} R^{\mu \nu \rho \sigma}-1740 \Lambda^{2},
\end{aligned}
$$

which clearly differs from the $M^{2}=0$ result

$$
\begin{aligned}
180(4 \pi)^{2} b_{4}^{(\Lambda)}(\text { total }) & \\
& =180(4 \pi)^{2}\left[b_{4}^{(\Lambda)}(1,1)-2 b_{4}^{(\Lambda)}(1 / 2,1 / 2)+b_{4}^{(\Lambda)}(0,0)\right] \\
& =212 R_{\mu \nu \rho \sigma} R^{\mu \nu \rho \sigma}-2088 \Lambda^{2} .
\end{aligned}
$$

(These one-loop differences between massive and massless spin 2 in the $\mathrm{A}=0$ case are well-known ${ }^{51}$ ). Even in the case of backgrounds with constant curvature

$$
\begin{aligned}
R_{\mu \nu \rho \sigma} & =\frac{1}{3} \Lambda\left(g_{\mu \nu} g_{\rho \sigma}-g_{\mu \rho} g_{\nu \sigma}\right), \\
R_{\mu \nu \rho \sigma} R^{\mu \nu \rho \sigma} & ={ }_{\partial} \mathrm{A}^{2},
\end{aligned}
$$

there is no cancellation. Thus we conclude that the absence of the discontinuity between the $M^{2}-\rightarrow 0$ and $M^{2}=0$ results for massive spin 2 , demonstrated in 
Ref. 45,44 , is an artifact of the tree approximation and that the discontinuity itself persists at one loop.

That the full quantum theory is discontinuous is not surprising considering the different degrees of freedom for the two cases. However, the three additional longitudinal degrees of freedom of the massive graviton do not couple to a conserved stress tensor. Thus, in the absence of any self-couplings (or at tree-level), the additional longitudinal modes would decouple from matter, yielding a smooth $M^{2} \rightarrow 0$ limit. Nevertheless, due to these self-couplings (seen here as couplings to the background metric in the linearized approach), these additional modes do not decouple, thus yielding the resulting discontinuity in the massless limit. (This result also suggests that the situation would be similar for the spin- $\frac{3}{2}$ case considered in Ref. 52,53.) Of course, these one loop effects are very small and so experiments such as the bending of light would still not be able to distinguish a massless graviton from a very light graviton in the presence of a non-zero cosmologocal constant.

Similarly, we note that a classical continuity but quantum discontinuity arises in the "partially massless" limit $M^{2} \rightarrow 2 \mathrm{~A} / 3^{54}$ as a result of going from five degrees of freedom to four ${ }^{50}$.

We finish with the important caveat that the $\mathrm{M} \rightarrow 0$ discontinuity for fixed $\mathrm{A}$ of the massless limit of massive spin-2 we have demonstrated applies to fields described by the Pauli-Fierz action discussed in Ref. 45,44. One may question whether this is a suitable action to describe the interaction of massive gravitons. We are not necessarily ruling out a smooth limit for other actions that might appear in KaluzaKlein or brane-world models, for example. Indeed one would expect a smooth limit if the mass is acquired spontaneously ${ }^{55}$ rather than through an explicit Pauli-Fierz term. In conventional Kaluza-Klein models, however, this limit, though smooth, would also be the decompactification limit and would result in massless gravitons in the higher dimension rather than four dimensions. A closer examination would be necessary to discern the form of the effective action describing the trapped graviton of the brane-world scenario of Refs. 56,57.

\section{Acknowledgments}

I am grateful to my collaborators Mirjam Cvetic, Fred Dilkes, James T. Liu, Hong Lu, Chris Pope, Wafic Sabra, Hisham Sati and Kelly Stelle.

\section{References}

1. J. H. Schwarz, Superstrings. The first fifteen years of super str ing theory, (World Scientific, 1985).

2. M. J. Duff, The world in eleven dimensions: supergravity, supermembranes and Mtheory, (IOP Publishing, 1999).

3. J. H. Schwarz, Recent progress in superstring theory, hep-th/0007130.

4. J. H. Schwarz, Does superstring theory have a conformally invariant limit?, hepth/0008009.

5. A. Achucarro, J. Evans, P. Townsend and D. Wiltshire, Super p-branes, Phys. Lett. 
B198, 441 (1987).

6. M. J. Duff, R. R. Khuri and J. X. Lu, String solitons, Phys. Rep. 259, 213 (1995), [hep-th/9412184].

7. G. T. Horowitz and A. Strominger, Black strings and p-branes, Nucl. Phys. B360 197 (1991).

8. M. J. Duff and J. X. Lu, The self-dual Type IIB superthreebrane, Phys. Lett. $\mathbf{B 2 7 3}$ 409 (1991).

9. J. Polchinski, Dirichlet-branes and Ramond-Ramondcharges, Phys. Rev. Lett. 75, 4724 (1995).

10. T. Banks, W. Fischler, S. H. Shenker and L. Susskind, M-theoryas a matrix model: $a$ conjecture, Phys. Rev. D55, 5112 (1997).

11. J. Maldacena, The large $N$ limit of superconformafield theories and supergravity, Adv. Theor. Math. Phys. 2, 231 (1998) [hep-th/9711200].

12. S. S. Gubser, I. R. Klebanov and A. M. Polyakov, Gauge theory correlators from noncritical string theory, Phys. Lett. B428, 105 (1998) [hep-th/9802109].

13. E. Witten, Anti-de Sitter space and holography, Adv. Theor. Math. Phys. 2, 253 (1998) [hep-th/9802150].

14. G. 't Hooft, Dimensional reduction in quantum gravity, gr-qc/9310026.

15. L. Susskind, The world as a hologram, J. Math. Phys. 36, 6377 (1995) [hep-th/9409089].

16. L. Randall and R. Sundrum, An alternative to compactification,Phys. Rev. Lett. 83, 4690 (1999) [hep-th/9906064].

17. L. Susskind and E. Witten, The Holographic Bound in Anti-de Sitter Space, hepth/9805114.

18. N. Arkani-Hamed, S. Dimopoulos and G. Dvali, The universe's unseen dimensions, Scientific American, August 2000, 62.

19. M. S. Bremer, M. J. Duff, H. Lü, C. N. Pope and K. S. Stelle, Instanton cosmology and domain walls from M-theory and string theory, NucL Phys. B543, 321 (1999) [hep-th/9807051].

20. M. J. Duff, J. T. Liu and K. S. Stelle, A supersymmetric Type IIB Randall-Sundrum realization, hep-th/0007120.

21. M. Cvetic, H. Lii and C.N. Pope, Localized gravity in the singular domain wall background?, hep-th/0002054.

22. S.P. de Alwis, Brane world scenarios and the cosmological constant, hep-th/0002174.

23. R. Kallosh and A. Linde, Supersymmetryand the brane world, JHEP 0002, 005 (2000) [hep-th/0001071].

24. K. Behrndt and M. Cvetic, Anti-de Sitter vacua of gauged supergravities with 8 supercharges, Phys. Rev. D61, 101901 (2000) [hep-th/0001159].

25. M. Cvetic, M. J. Duff, J. T. Liu, H. Lu, C. N. Pope and K. S. Stelle, Randall-Sundrum brane tensions, hep-th/0011167.

26. P. Krauss, Dynamics of anti-de Sitter domain walls, JHEP 9912, 011 (1999) [hepth/9910149].

27. T. Ghergetta and A. Pomarol, Bulk fields and supersymmetry in a slice of AdS, hepth/0003129.

28. N. Alonso-Alberca, P. Meessen and T. Ortin, Supersymmetric brane-worlds, Phys. Lett. B482, 400 (2000) [hep-th/0003248].

29. E. Bergshoeff, R. Kallosh and A. Van Proeyen, Supersymmetry in singular spaces, JHEP 0010, 033 (2000) [hep-th/0007044].

30. J. T. Liu and H. Sati, Breathing mode compactificationsand supersymmetry of the brane world, hep-th/0009184

31. R. Altendorfer, J. Bagger and D. Nemechansky, Supersymmetric Randall-Sundrum scenario, hep-th/0003117. 
32. M.J. Duff, James T. Liu and W.A. Sabra, Localization of supergravity on the brane, hep-th/0009212.

33. H. Lu and C. Pope, Branes on the Brane, hep-th/0008050.

34. B. Bajc and G. Gabadadze, Localization of matter and cosmological constant on a brane in anti de Sitter space, Phys. Lett. B474, 282 (2000) [hep-th/9912232].

35. A. Pomarol, Grand unified theories without the desert, hep-th/0052931

36. N. Kaloper, E. Silverstein and L. Susskind, Gauge Symmetry and Localized Gravity in $M$ Theory, hep-th/0006192.

37. M.J. Duff and James T. Liu Hodge duality on the brane, hep-th/0010171.

38. J. Maldacena, Supergravity description offield theories on curved manifolds and a no-go theorem, hep-th/0006085.

39. M. J. Duff, Problems in the classical and quantum theories of gravitation, Ph. D. thesis, Imperial College, London (1972).

40. M. J. Duff, Quantum corrections to the Schwarzschildsolution, Phys. Rev. D9, 1837 (1974).

41. M. J. Duff and James T. Liu, Complementarity of the Maldacena and Randall-Sundrum pictures, Phys. Rev. Lett. 85, 2052 (2000) [hep-th/0003237].

42. H. van Dam and M. Veltman, Massive and massless Yang-Millsand gravitationalfields Nucl. Phys. B22, 397 (1970).

43. V.I. Zakharov, JETP Lett. 12, 312 (1970).

44. I. I. Kogan, S. Mouslopoulos and A. Papazoglou, The $m \rightarrow 0$ limitfor massive graviton in $d S_{4}$ and $A d S_{4}$ : How to circumvent the van Dam-Veltman-Zakharov discontinuity hep-th/0011138.

45. M. Porrati, No van Dam-Veltman-Zakharovdiscontinuity in AdS space hep-th/0011152.

46. A. Higuchi, Forbidden Mass Range For Spin-2 Field Theory In De Sitter Space-Time Nucl. Phys. B282, 397 (1987).

47. A. Higuchi, Massive symmetric tensor field in space-times with a positive cosmological constant Nucl. Phys. B325, 745 (1989).

48. F. Dilkes, M. J. Duff, James T. Liu and H. Sati, Quantum discontinuity between massless and infinitessimalgraviton mass with a A term Phys. Rev. Lett. (to appear), hepth/0102093.

49. S. M. Christensen and M. J. Duff, Quantizing gravity with a cosmological constant Nucl. Phys. B170, 480 (1980).

50. S. Deser and R. I. Nepomechie, Gauge Invariance Versus Masslessness In De Sitter Space Annals Phys. 154, 396 (1984).

51. S. M. Christensen and M. J. Duff, Axial and conformal anomalies for arbitrary spin in gravity and supergravity Phys. Lett. 76B, 571 (1978).

52. P. A. Grassi and P. van Nieuwenhuizen, No van Dam-Veltman-Zakharov discontinuity for supergravity in AdS space hep-th/0011278.

53. S. Deser and A. Waldron, Discontinuities of massless limits in spin 3/2 mediated interactions and cosmological supergravityhep-th/0012014.

54. M.J. Duff, James T. Liu and H. Sati, Quantum $M^{2} \rightarrow 2 \mathrm{~A} / 3$ discontinuity for massive gravity with a Lambda term, hep-th/0105008.

55. M. J. Duff, Dynamical breaking of general covariance and massive spin-two mesons Phys. Rev. D12, 3969 (1975).

56. I. I. Kogan, S. Mouslopoulos and A. Papazoglou, A new bigravity model with exclusively positive branes Phys. Lett. B501, 140 (2001) [hep-th/0011141].

57. A. Karch and L. Randall, Locally localized gravity hep-th/0011156. 